\title{
Some aspects of continuous patient monitoring
}

\author{
H. S. WolfF \\ B.Sc. \\ Division of Biomedical Engineering, \\ National Institute for Medical Research, Hampstead, London, N.W.3
}

Patient monitoring equipment has now been commercially available for about 10 years. Large sums of money have been spent in some instances on elaborate installations, initially described with enthusiasm by their owners but often found to be considerably under utilized in practice. The author, and a team in his laboratory, have been responsible for designing a modular patient monitoring system which is currently being commercially developed. At the same time trials have been conducted in a number of hospitals to investigate the contribution which monitoring can make in different clinical situations. Initially the situation tended to be dominated by technical failures, and it was thought that once these had been largely eliminated the equipment would be used with enthusiasm.

However, whilst technical improvement was undoubtedly appreciated, it was also discovered that even the best technology was not good enough by itself to overcome psychological barriers on the part of nurses and doctors, lack of elementary appreciation of the treatment and needs of instruments. There was a lack of understanding of the significance of changes in magnitude of physiological measurements. The previous sentence which sounds like an accusation is not meant as such, it merely indicates that on one hand the equipment designer must have a clear understanding of the motivation and state of technical competence of the users of his equipment, and design accordingly. On the other hand the user organization must accept that the purchase of equipment must be accompanied by a deliberate decision to raise the level of 'technological culture' amongst the staff, if the full value is to be obtained from the investment.

When equipment is bought, the assumption is made that the acquisition of physiological information by automatic means from patients undergoing surgery, in intensive care, in recovery rooms, and in corcnary care situations is capable of being of clinical value and can lead to better patient care. To these situations can be added those of premature infants and of mothers in labour who may require apparatus which may be different in some respects, but where the same general principles apply.

Because of inherent tensions and stresses which these situations generate, equipment only becomes $\vec{O}$ acceptable if the following three conditions are met: $\vec{\overrightarrow{ }}$

1. At the time at which the equipment is put intoo use on the sick patient, the mere fact of activating ifo should not make any appreciable conscious demands 3 on the staff.

2. The overall reliability should be such that in: the mind of the user there is no serious doubt that the $\vec{\omega}$ results which are displayed represent the true state of $\vec{N}$ affairs, or that the results are at least as good as those which would have been obtained using traditional ${ }^{\circ}$ methods.

3. The measurements are not only correct and reliable but also make a contribution to the clinical management of the patient.

The future of any system of patient monitoriag $\vec{\omega}$ must be examined from these points of view. Startier $\searrow$ with (3) I would have thought that even the prese repertoire of:

(a) heart rate from the ECG and display of ECG wave-form;

(b) direct intra-arterial blood pressure; display of $\frac{\alpha}{\mathrm{O}}$ the arterial pressure wave-form and pulse rate from arterial pressure wave-form;

(c) indirect measurement of blood pressure in suitable cases;

(d) central venous pressure;

(e) temperature at various body sites;

(f) respiration rate in some patients provided that conditions (1) and (2) are also met, is $\overline{3}$ capable of making a contribution to the clinical management of the patient.

I would also maintain that the methods which are available allow measurement to be performed by trained staff with a reliability which is comparable with that obtained by manual procedures. However, even if the methods are adequate there are still $\%$ occasional technical breakdowns, which though $N$ they have decreased markedly in modern equipment $N$ still make it difficult to completely fulfil condition (2).

I see the future development of monitoring equipment, commercial considerations aside, proceeding along the following lines: There has to be a radical $\stackrel{\infty}{\Phi}$ change in the approach to the training of operating staff (Fig. 1).

Comparisons with the operation of television sets $\frac{\mathrm{d}}{\mathbb{Q}}$ 


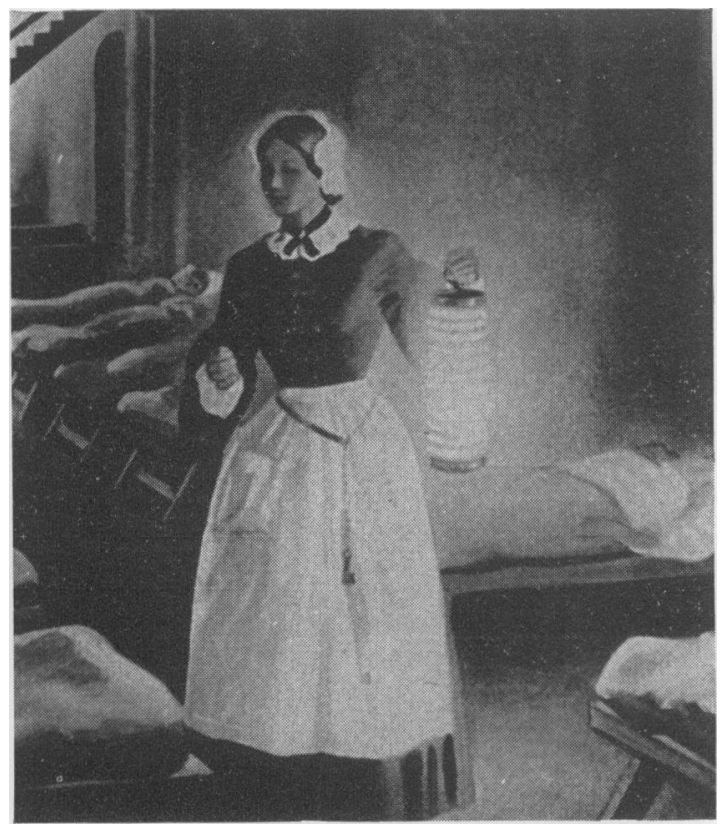

Fug. 1. There is practically no technological element in a nurse's training course and few hospitals have any facilities for giving training beyond teaching the operation of a given instrument. At present even the refresher courses for Sister Tutors contain little material on instrumentation.

and motor cars and even radiography are facile because in none of these cases is a strictly quantitative result required. In monitoring, the validity of the procedure will usually be judged explicitly by the accuracy of the measurement, however defined. To achieve an acceptable level, and to recognize the conditions when this level cannot be achieved, does require training specific to the activity of monitoring. The reasons for the popularity of the ECG displays are not only that it is a useful piece of information, but also that the wave-form does not have to meet any exact quantitative standards, and moreover there is no 'manual' and presumed correct method for comparison. It is impossible to meet condition (1) unless it is recognized that, even with technically completely reliable equipment, it cannot perform its functions unless nursing and medical staff achieve a degree of familiarity in its management which makes its use in an emergency situation second nature, not requiring reference to third parties, instruction books, or trial-and-error procedures. To the best of my knowledge, this state of affairs has not been achieved completely in any hospital, and until it has, patient monitoring cannot succeed (Fig. 2).

The amount and quality of training does not have to be very great, but it must involve the opportunity to practice in situations where there is no clinical

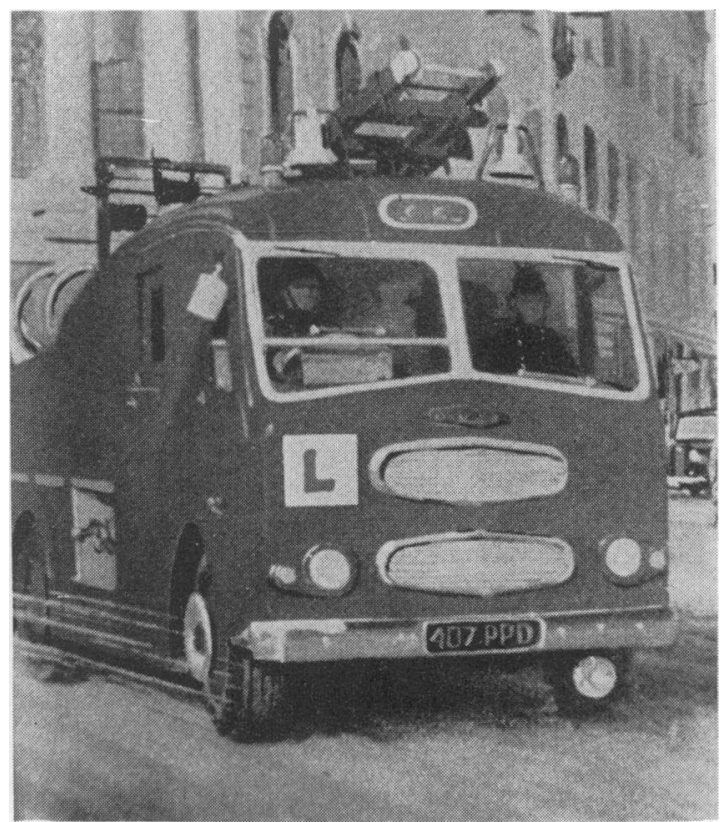

Flg. 2. Little opportunity is given to nurses even in intensive-care units to use equipment under training conditions. This is rather like only teaching fire engine drivers to drive whilst actually on the way to a fire.

pressure and where questions can be asked and mistakes made without compromising the treatment of a patient. It must also include an understanding of how the measurements are made, how the procedure differs from the comparable manual method, and of the importance(or lack of importance) of difference between monitored values and nursing measurements.

To achieve these ends means formal courses and $a$ degree of stability in the staff of units where equipment is used. The manufacturer, initially in conjunction with specialist hospital staff should provide the courses; the hospital will have to be prepared to release staff periodically for 1 or 2 days, to pay for the courses either directly, or else as part of the purchase price or service agreement and to undertake to limit the turnover of staff in intensive or coronary care units so as to maintain a reasonable concentration of trained staff.

The hospital administration also has its part to play. It must be realized that monitoring equipment is in principle no different from any other capital plant. It depreciates with time, financial provisions have to be made for its eventual replacement, repair costs will mount as the equipment becomes older, and a running budget must be available to cover the cost of expendable stores and minor replacements (Fig. 3).

If adequate training is given, the quality of the results, and hence the confidence with which they are 


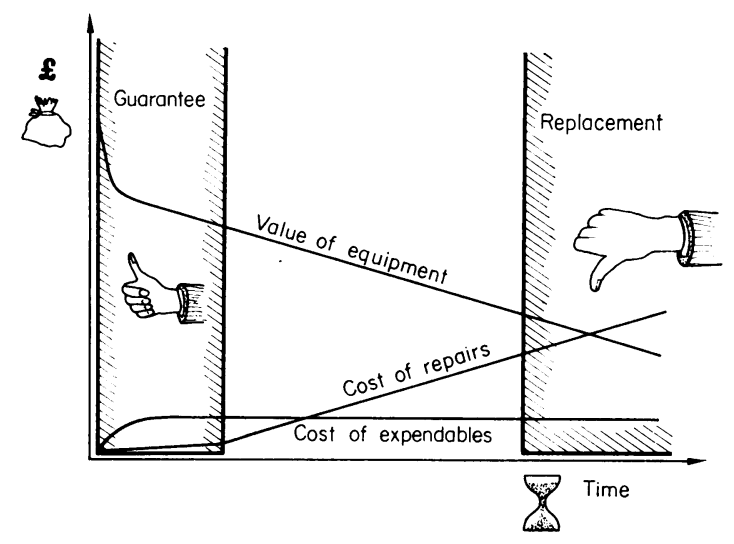

FIG. 3. The value of equipment decreases from the moment it is bought, both in terms of the amount of maintenance required and for reasons of obsolescence. At some point further operation becomes uneconomic and money will have to be made available for replacement.

regarded will improve. There are still technical problems and these can again be divided into three categories:

(1) An inherent defect in the method of measurement. For instance, at present respiration rate measurement is unsatisfactory in conscious patients, and an improved method will have to be developed.

(2) Technical failures which, whilst real in the sense that something has become broken or deranged, are in principle no more serious than a blocked hypodermic needle, and can be cured in the same way by replacement of the defective component. Some types of equipment have been specifically designed to make replacement of modules without tools easy, and the problem is thus reduced to one of identifying which part to replace (Fig. 4). Failures of this nature are almost inevitable, but here again an almost trivial amount of training combined with printed fault-finding charts will allow the staff to clear such faults with no more trouble than replacing the blocked needle or the kinked catheter (Fig. 5).

(3) The use of a method of measurement which is inherently unsuitable for the situation in which it is applied. For instance, indirect blood pressure measurement cannot be applied in its automatic form in patients who are restless and wave their arms about. This must be realized and the method must either be used in a 'demand' mode under nursing supervision, which is still less tedious than employing traditional means, or the intra-arterial method must be employed. If it could be shown (as I believe that it has) that the insertion of a fine catheter into a comparatively small artery like the radial or brachial artery is attended by very little risk even if left in place for some days, then the reliability of several measurements could be improved dramatically. The

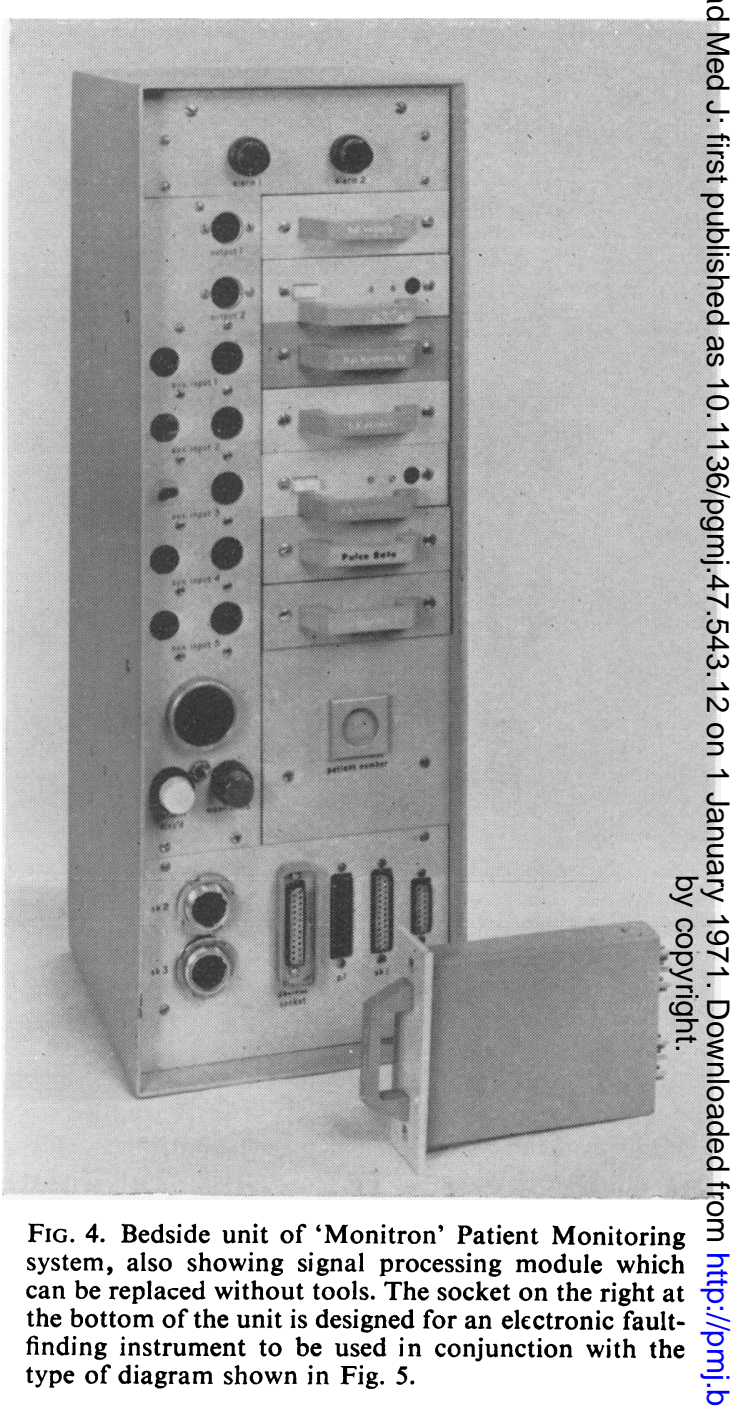

requirements are a transducer and its associated. electronics which are substantially free from drif and a catheter-flushing system which operates con tinuously and requires no nursing supervision. The first requirement can be met by appropriate selections of existing equipment, the second by the use of a pressurized bag of flushing fluid and a suitablow hydraulic resistance or by the 'Delta' pump shown in' Fig. 6. The pump has the advantage that the volumes delivered is known and independent of the bloodew pressure of the patient. Once an intra-arterial cathe ter is in place it will provide a highway for reliableo data, not only for systolic and diastolic bloodळ pressure, but also for 'real' pulse rate, and in the? foreseeable future for oxygen tension.

Training must lead to an understanding of what is being done, and a realization that the machine is in 
FAULT - INCORRECT PULSE RATE READING

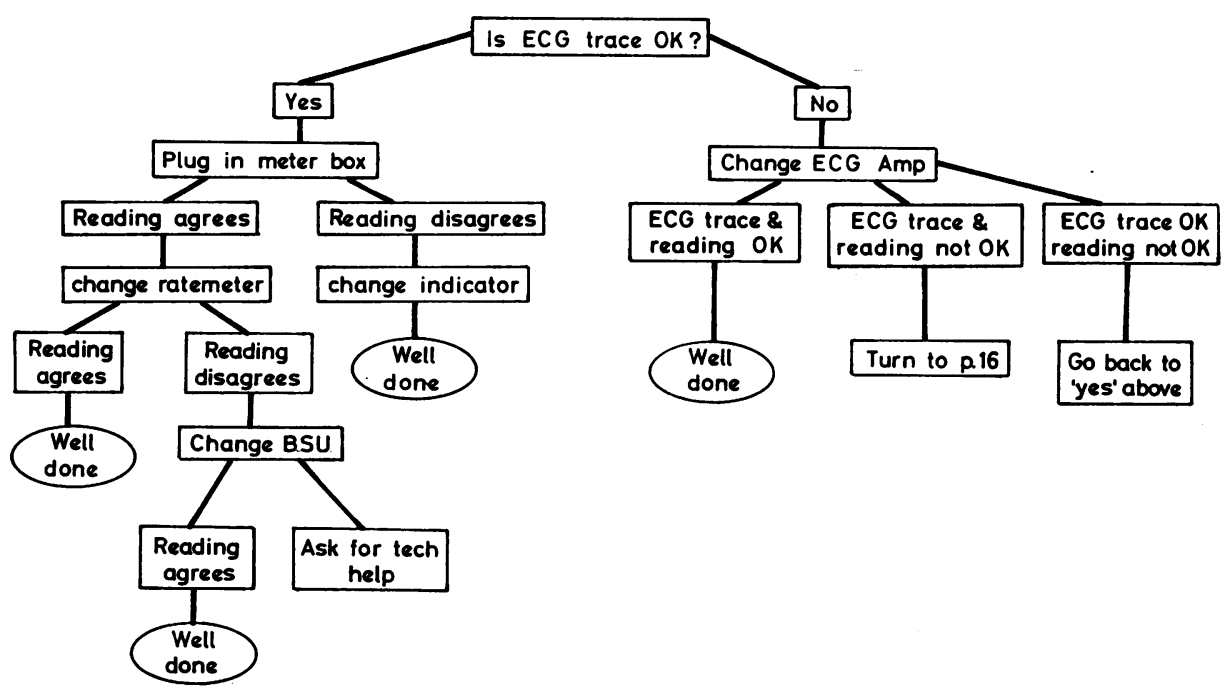

FIG. 5. Fault finding chart intended to be used by nurses in conjunction with specially designed, very simple test instruments which can be plugged into "diąnostic sockets' on the monitron units. No tools or special knowledge are required. What is required is a stcck of spare modules.

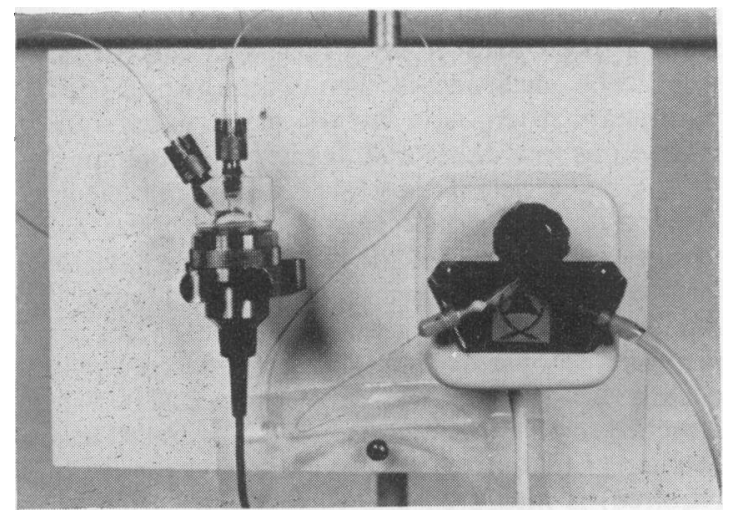

Fig. 6. 'Delta' pump and strain gauge blood pressure transducer. A long coil of fine nylon tubing isolates the compliance of the pump from the gauge. All tubing parts including the pump are disposable.

general no better than the nurse or doctor in its ability to elicit a measurement, it is merely more consistent, does not get tired, and removes the drudgery of monotonously repeated procedures.

\section{The development of new transducers and methods of signal analysis}

Items which are known to be under development are: (a) Off-line but automatic blood gas analysis equipment $\left(\mathrm{pH}, \mathrm{PCO}_{2}, \mathrm{Po}_{2}\right)$. This instrument will require no skill for its operation and will wash and calibrate itself completely automatically.

(b) An intra-vascular $\mathrm{Po}_{2}$ catheter tip electrode only $0.6 \mathrm{~mm}$ in diameter. This could well become a disposable item.

(c) A method of continuous central venous pressure measurement with automatic compensation for relative movement between the patient and the transducer. Such development is essential if longterm continuous central venous pressure measurement is to be performed.

(d) Methods of ECG analysis directed specifically towards the detection of arrhythmias.

(e) The reduction of false alarms by: (1) the detection of instrument failures without sounding the alarm; (2) the logic combination of more than one deviation from normality before an alarm is sounded.

Monitoring is moving out of the phase where its novelty was sufficient to generate enthusiasm, into one where its cost and the benefits which result are being compared in a cool and rational manner. In my opinion in the immediate future it is the provision of an appropriate technological climate in the hospital which will determine the benefits rather than major technological developments. 